\title{
Ge quantum dots sandwiched between two thick Si blocking layers to block the dark current and tune the responsivity spectrum
}

\author{
Y.H. Peng ${ }^{\text {a }}$, C.C. Chen ${ }^{\text {a }}$, C.H. Kuan ${ }^{\mathrm{a}, *}$, H.H. Cheng ${ }^{\mathrm{b}}$ \\ a Department of Electrical Engineering, Graduate Institute of Electronics Engineering, National Taiwan University, \\ No. 1, Sec. 4, Roosevelt Road, Taipei 106, Taiwan, ROC \\ ${ }^{\mathrm{b}}$ Center for Condensed Matter Sciences, National Taiwan University, Taipei 10617, Taiwan, ROC
}

Received 7 January 2003; accepted 12 March 2003

\begin{abstract}
A p-type Ge quantum dot infrared photodetector is investigated in this paper. The intrinsic thick Si layers are utilized to block the dark current under low bias and adjust the tunneling probability by controlling applied field to tune the responsivity spectrum. The $30 \mathrm{~K}$ responsivity spectrum shows broadband from 2 to $9 \mu \mathrm{m}$ at zero bias with photovoltaic effect and extends its range to $20 \mu \mathrm{m}$ when the applied bias is increased.

For long wavelength detection, our detector is tunable with Si blocking layer. The detectivity in this sample has maximum value toward $2.1 \times 10^{10} \mathrm{~cm}\left(\mathrm{~Hz}^{0.5}\right) / \mathrm{W}$ under $0.2 \mathrm{~V}$ for the $6 \mu \mathrm{m}$ wavelength. That for the long wavelength is $\sim 10^{9} \mathrm{~cm}\left(\mathrm{~Hz}^{0.5}\right) / \mathrm{W}$.

(c) 2003 Elsevier Ltd. All rights reserved.
\end{abstract}

Keywords: Ge quantum dot infrared photodetector; Long wavelength infrared photodetector; Blocking layer; Wavelength tunability

\section{Introduction}

Quantum well infrared photodetector (QWIP) are useful in various applications such as the thermal imaging, night vision, and optical communication etc. [1-7]. Recently, the detectors realized with quantum-dot structure are particularly worthy of academic investigation because the quantum dot is a nanostructure. Due to the limitation of the polarization selection rule, QWIP will have intersubband transition only by the light polarized in the growth direction, while the quantum dot's three-dimensional confinement potential for charge carriers makes the quantum dot insensitive to the polarization direction [8-11,20]. This characteristic can make the fabrication process easier.

\footnotetext{
*Corresponding author. Tel.: +886-2-23635251x439; fax: +886-2-23671909.

E-mail address: kuan@cc.ee.ntu.edu.tw (C.H. Kuan).
}

Although quantum dot infrared photodetectors (QDIPs) are predicted to have some important advantages over QWIPs such as lower dark current, higher internal gain and higher operation temperature $[12,13]$, the practical detector in fact does not show the advantages. Especially some structures are needed to limit the dark current in order not to saturate the read-out circuit. In addition, Ge quantum dots (QDs) have become a hot research topic recently since this Si-based detector has the advantage of the monolithic integration with the read-out circuit [14].

In order to cut down the dark current, the detector is realized with Ge QDs that are sandwiched between two thick Si blocking layers. Since the grown Ge QDs have different sizes, the resulting excited energy states may have a broad range and the detected wavelength can then be wide. For the long wavelength detection, the energy states of the photoholes may be close to the top of the quantum dot barrier [15-21]. The two thick blocking layers can be used to adjust the electric field 
around the barrier. Under the low bias, the photoholes cannot pass the barrier. However, under high bias, the effective thickness of the barrier becomes thin due to the strong electric field. The photoholes may hence tunnel through the barrier and the apparent responsivity for the long wavelength can then be detected [22]. By manipulating the thickness of the blocking layers and quantum dot's size, we can broaden the detection range to longer wavelength and make the detector tunable in the long wavelength range.

\section{Investigation of the sample structure}

First of all, we grew a $7 \mathrm{ml} \mathrm{Ge}$ QDs on a Si substrate with well-grown buffer at the temperature of $600{ }^{\circ} \mathrm{C}$, and observed the size, shape, and distribution density with an atomic force microscope (AFM) and the experimental results are shown in Fig. 1. For the QDs as marked by the line in Fig. 1(a), the associated cross section view is shown in Fig. 1(b). From the results, we could measure the height and diameter of Ge QDs to be around 200-300 А, and $2500 \AA$ respectively. From the AFM picture, the calculated distribution density of $\mathrm{Ge}$ QDs is around $1.3 \times 10^{19} \mathrm{~cm}^{-2}$.

Fig. 2 shows the structure of our photodetector. The growth procedure was first to deposit a $3000 \AA$ Si buffer on p-type 1-10 $\Omega \mathrm{cm}$, which was doped with boron of $10^{19} \mathrm{~cm}^{-3}$, to serve as the bottom contact. After that we deposited a $1000 \AA$ Andoped Si blocking layer with $2 \times 10^{16} \mathrm{~cm}^{-3}$ background doping. Then 10 layers of multiple quantum dots (MQDs) were deposited. The growing parameter of MQDs was derived from AFM data. We deposited $7 \mathrm{ml} \mathrm{Ge}$ QDs, and added delta dope

(a)
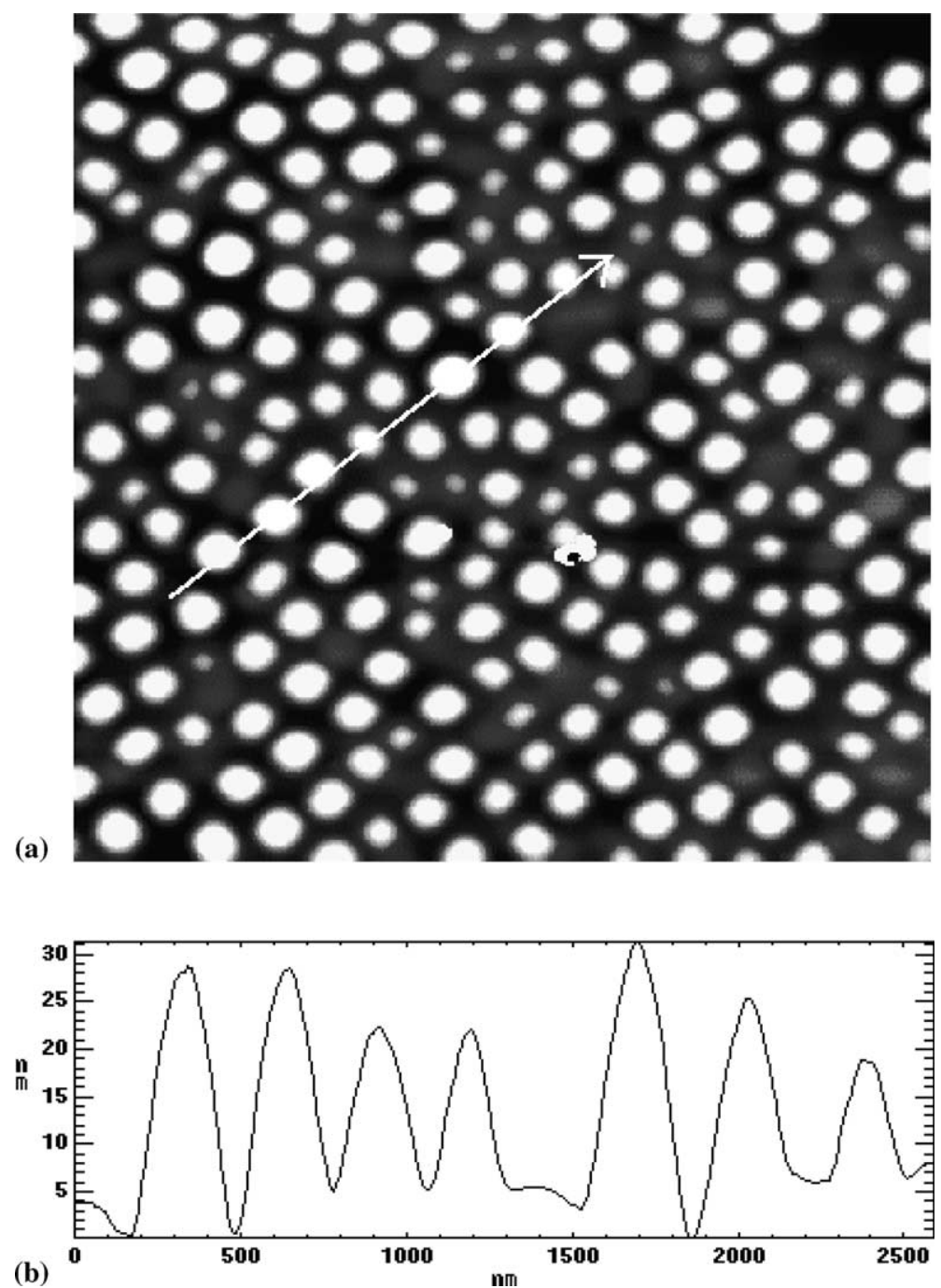

Fig. 1. AFM picture of pre-growth surface QDs (a) and its cross section plot (b). 


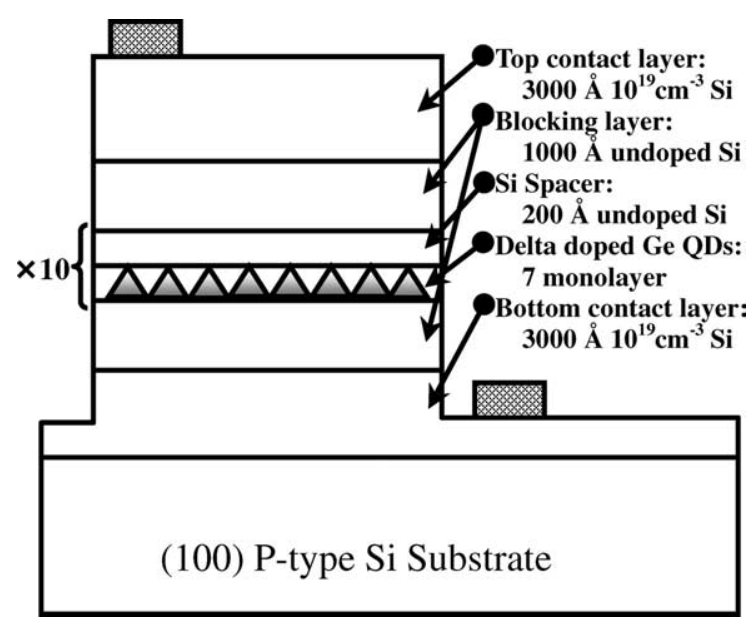

Fig. 2. The structure of our quantum well infrared photodetector.

in the middle of the Ge QDs. After that, 200- $\AA$ undoped $\mathrm{Si}$ was caped on these QDs as spacer to smoothen the surface roughness that resulted from the Ge QDs. This spacer also serves as a barrier on the energy band in order to form the quantum confinement to the hole of Ge QDs. After the MQDs came the 1000- $\AA$ undoped $\mathrm{Si}$ blocking layer and then the top contact layer with 3000 A thick and $1 \times 10^{19} \mathrm{~cm}^{-3}$ boron doped.

The whole structure was then examined by highresolution cross-section TEM. The result is shown in Fig. 3. The width and size of the MQDs are about 200 and $1400 \AA$ respectively. The TEM picture also shows the vertical alignment of the MQDs layer, which is resulted from strain. In addition to this, a phenomenon similar to Newton Ring is also found around the QDs in the picture, which reveals that there are strong strain field in the MQDs [23].

We also etched the heavily doped layer on the surface, and did the photoluminescence (PL) measurement at the temperature of $16 \mathrm{~K}$. The result of this measurement is shown in Fig. 4(a). The PL spectra at 1.14, 1.098 and $1.036 \mathrm{eV}$ are the phonon assisted transitions of TA, TO,

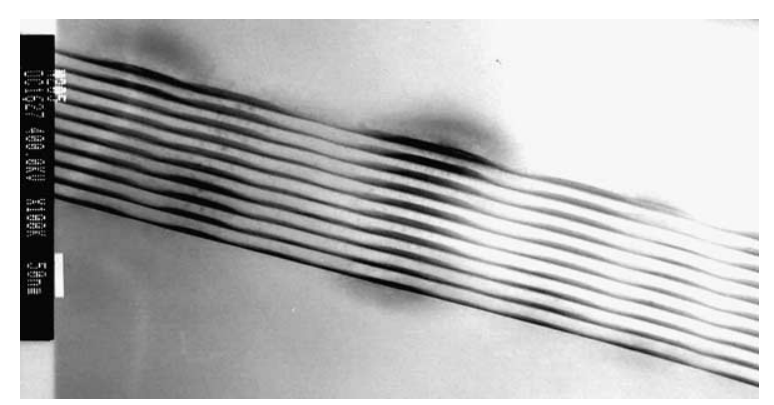

Fig. 3. Cross section TEM of QDIP.
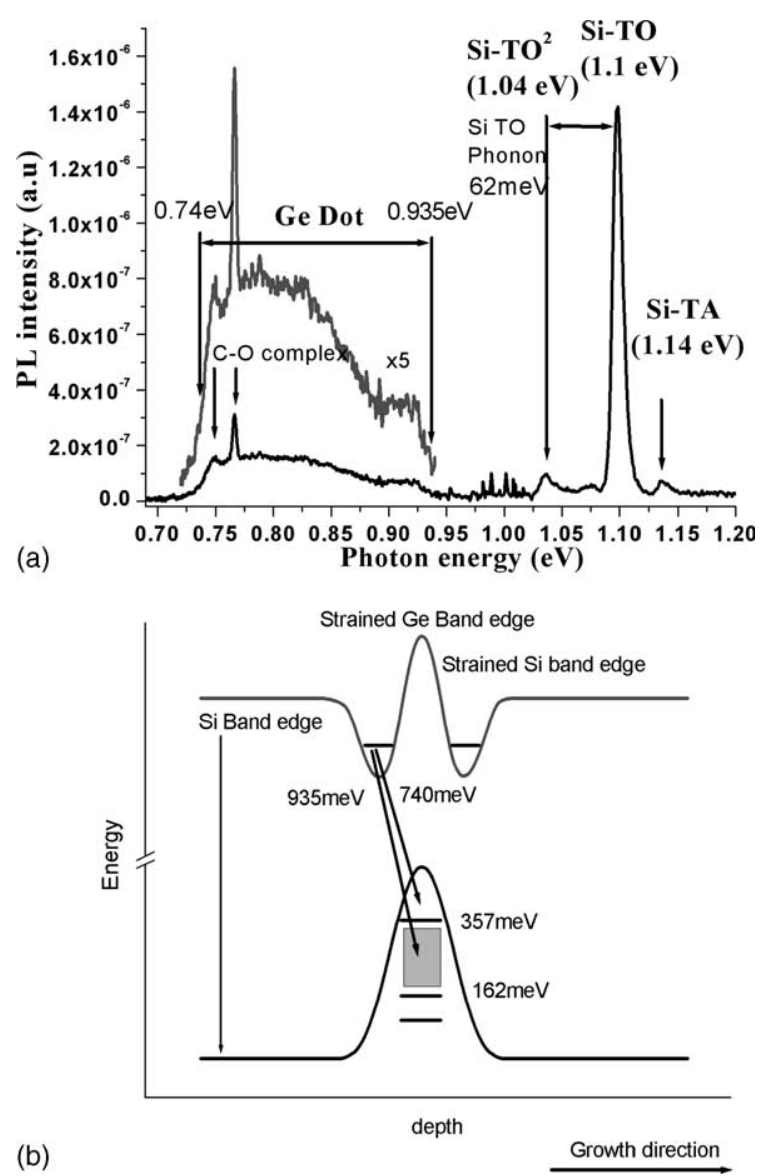

(b)

Growth direction

Fig. 4. (a) PL spectrum at $20 \mathrm{~K}$ temperature; and (b) schematic energy band diagram according to PL spectrum (not to scale).

and $\mathrm{TO}_{2}$ of Si respectively. From the discrepancy of TO and $\mathrm{TO}_{2}$, it is concluded that the energy of Si TO phonon is $62 \mathrm{meV}$. From the $\mathrm{Si}-\mathrm{TO}$ transition energy, it is then concluded that the energy of Si band gap is $1.16 \mathrm{eV}$. In addition, the transition peaks at 0.75 and $0.766 \mathrm{eV}$ are related to $\mathrm{C}-\mathrm{O}$ complex and mainly come from the spectrum of substrate [24].

The range from 740 to $935 \mathrm{meV}$ in Fig. 4(a) is the transition formed by Ge QDs and indicates the QD's size distribution. According to the neighboring confinement structure proposed by the Refs. [25-28], the transition of the Ge related PL is primarily from the localized state near the Si conduction band at the inplane $\mathrm{Si} / \mathrm{Ge}$ hetero interface to the ground state in the valence band of Ge dots as shown in Fig. 4(b). It originates from spatially indirect and phononless (NP) recombination between holes confined in the Ge dots and electrons confined within the tensile strained $\mathrm{Si}$ encompassing the coherent Ge dots. In addition, the broadness of the Ge related PL might be attributed to the size distribution of the QDs, which results in a band of the 
QDs. Therefore, the levels of Ge QD ground-state band can be estimated by Ge phononless transition energy and the activation energy from the tensile strained $\mathrm{Si}$. Suppose $63 \mathrm{meV}$ be adopted for the activation energy [25] and the energy origin be the silicon valence band edge. The uppermost and lowermost level of the groundstate band in the Ge dots can be calculated as 162 and $357 \mathrm{meV}$ respectively as shown in Fig. 4(b).

A standard process of the detector fabrication was also performed on the sample. A mesa of $300 \times 600 \mu \mathrm{m}^{2}$ protruding to the middle of bottom contact layer was etched. When $1500 \AA \mathrm{Al}$ was deposited at the top and bottom of the mesa, the rapid thermal annealing proceeded immediately to form ohmic contacts.

\section{Characterization of our detector}

The $I-V$ characteristics of the detector were measured to analyze its electric property. The dark current (solid curves) versus biases at different temperatures and background photocurrent (dashed curves) at $20 \mathrm{~K}$ are showed in Fig. 5. The dark current at $20 \mathrm{~K}$ is extremely low compared with conventional QDIP. At low bias, the dark current is lower than exceed $10^{-11} \mathrm{~A}$, and appears to be turned on at $1.2 \mathrm{~V}$ bias.

The associated turn-on voltage decreases with increasing temperature. From 20 to $40 \mathrm{~K}$, the turn-on bias decreases from 1.2 to $0.5 \mathrm{~V}$, and decreases to zero at 50 $\mathrm{K}$, i.e. there is no turn-on phenomena above $50 \mathrm{~K}$. Similar situations occur when negative biases are applied. On the other hand, there is no turn-on phenomenon on the background photocurrent but there is a photovoltaic effect at zero bias. It indicates that the background photocurrent does not encounter any bar-

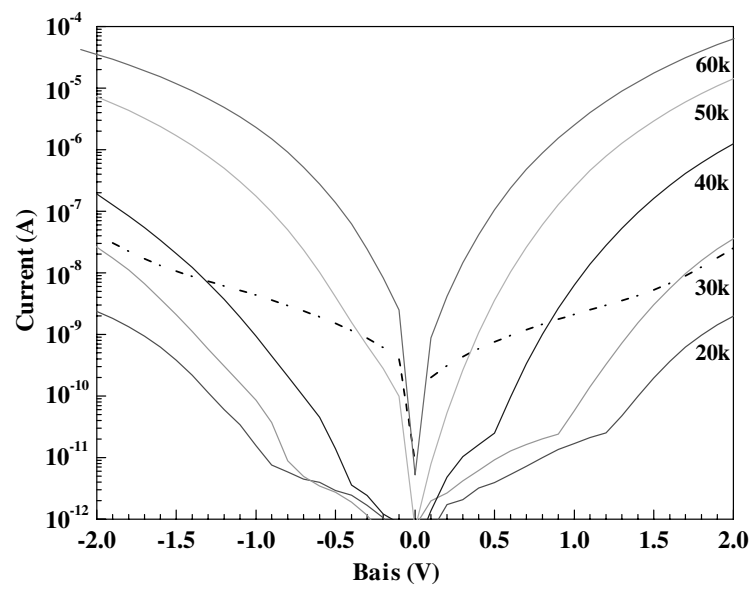

Fig. 5. $I-V$ curves for QDIP. The solid lines are dark current at $20-60 \mathrm{~K}$ temperatures. The dash line is photocurrent at $20 \mathrm{~K}$ temperature. rier [29]. When we compare it with the dark current at 20 $\mathrm{K}$, it is found that the background photocurrent is $10^{3}$ larger than the dark current under $1.2 \mathrm{~V}$ bias.

The dark current under low bias is attributed to the thermionic emission of holes after turning on; the current is dominated by the thermally assisted tunneling [30]. From the difference of dark and photocurrent level, we estimate the background-limited performance is toward $40{ }^{\circ} \mathrm{K}$ when we applied bias lower than $0.7 \mathrm{~V}$.

From Fig. 5, it is noted that the dark-current level is reduced obviously by two thick $\mathrm{Si}$ blocking layers. Suppose that the maximum voltage of the read-out circuit in the thermal imaging application is $5 \mathrm{~V}$ and the charge capacitance with the same area as our detector is $1 \mathrm{nF}$. If the reading time is $30 \mathrm{~ms}$ to reach low noise, then the maximum dark current is about $0.17 \mu \mathrm{A}$. If the operating temperature of our detector is $50 \mathrm{~K}$, then the applied bias should be less than $0.5 \mathrm{~V}$ in order not to saturate the capacitance. If the detector is to operate in the background limited performance, the applied bias has to be less than $0.3 \mathrm{~V}$.

The responsivity versus wavelength at $30 \mathrm{~K}$ for positive biases is shown in Fig. 6. The responsivity broadband of our QDIP at zero bias ranges from 1.6 to $9 \mu \mathrm{m}$. With the increase of bias, the spectrum gradually reaches over $20 \mu \mathrm{m}$ at $1.0 \mathrm{~V}$ bias. At zero bias, the spectrum shows that only photons with energy over $9 \mu \mathrm{m}$ can trespass the barrier and then be collected by the contact layer to form photocurrent. It is the photovoltaic effect as described in previous paragraph. While the bias increases, the spectrum extends from $9 \mu \mathrm{m}$ toward longer wavelength and the responsivity for the long wavelength hence increases. Under $1.0 \mathrm{~V}$ bias, it can be extended to be longer than $20 \mu \mathrm{m}$.

Fig. 6 indicates that there are involved with two mechanisms for the photohole to be emitted over and

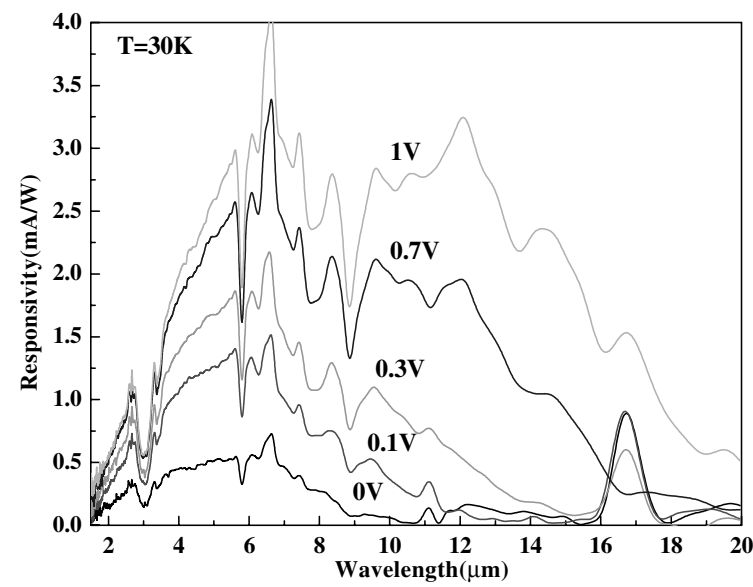

Fig. 6. Response spectrums at $20 \mathrm{~K}$ temperature with various bias form $0-1 \mathrm{~V}$. 
tunnel through the barrier of the QDs. The key wavelength to separate these two mechanisms is about $9 \mu \mathrm{m}$. The photoholes excited by the photons with wavelength less than $9 \mu \mathrm{m}$ have enough energy to be emitted over the barrier so that the photovoltaic effect appears at zero bias [29]. On the other hand, the photoholes excited by longer wavelength does not have enough energy and have to tunnel through the barrier with the help of the strong electric field so that the responsivity in Fig. 6 extends to longer wavelengths and shows the advantage of the tunable spectrum under the applied bias.

Since the band offset of Ge QDs and Si on the valence band is very large, it is expected that there would be at least two bands of energy states in the QDs [31]. From the photovoltaic effect in Fig. 6, it is concluded that the depth of the ground-state band is $138 \mathrm{meV}$, which is close to $162 \mathrm{meV}$ derived from PL and shown in Fig. 4(b). The discrepancy depends on the choice of the cutoff wavelength of the photovoltaic effect. If $8 \mu \mathrm{m}$ is chosen, the agreement between the responsivity and PL becomes obvious. From the longest wavelength of responsivity, we can determine the gap between the lowest level of the second band in QDs and the silicon barrier. Since the responsivity is cut off at $62 \mathrm{meV}$, the gap for the second-band bottom state to the Si barrier is about $100 \mathrm{meV}$. Since the value is not very large, it is possible for the associated photoholes to tunnel through under enough bias. To demonstrate the relation about the transition energies, a simplified band diagram for $\mathrm{Ge}$ QDs is showed in Fig. 8.

The specific detectivity for different wavelengths versus bias at $30 \mathrm{~K}$ is shown in Fig. 7. The detectivity in this device has maximum value toward $2.1 \times 10^{10} \mathrm{~cm}\left(\mathrm{~Hz}^{0.5}\right) / \mathrm{W}$

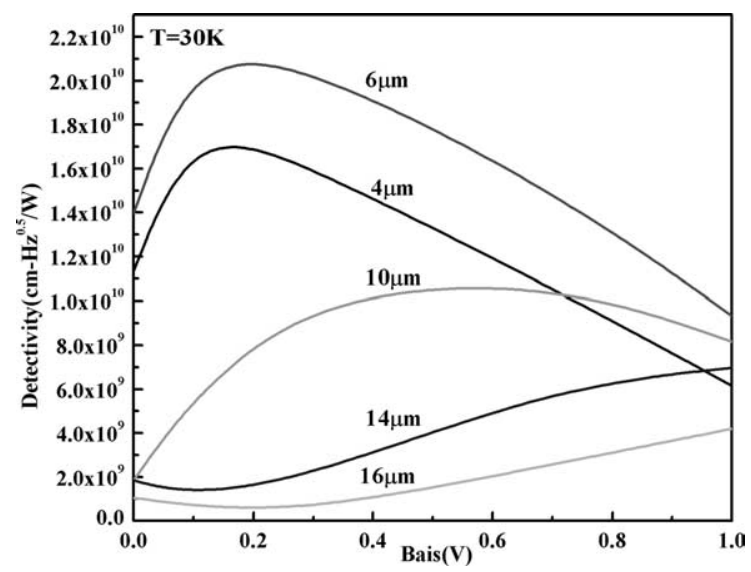

Fig. 7. Different wavelength detectivity under $0-1.0 \mathrm{~V}$ bias. There are two behaviors for these curves; one has its maximum under $0.2 \mathrm{~V}$ and decrease while bias increasing. The other is increasing while bias increasing. The two types of these curves are divided by $10 \mu \mathrm{m}$ curves.

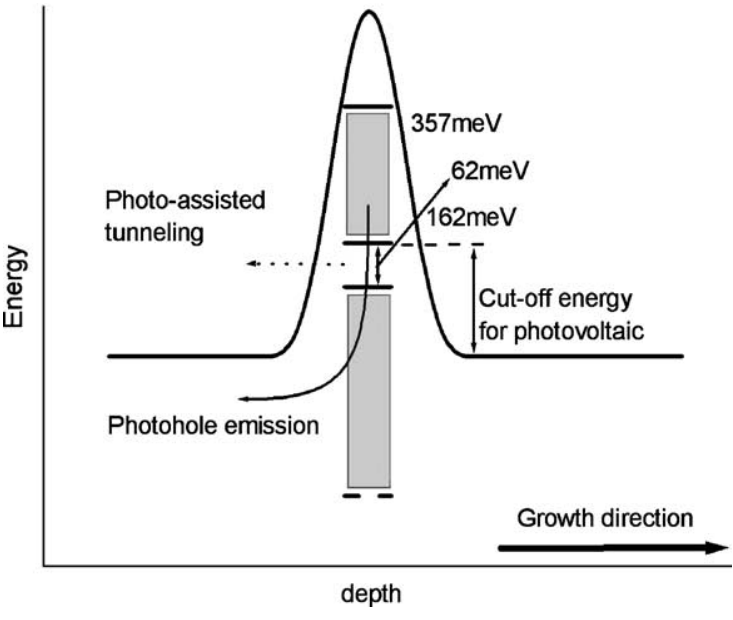

Fig. 8. The simplified band diagram for QDs in QDIP.

under $0.2 \mathrm{~V}$ for the $6 \mu \mathrm{m}$ wavelength. The detectivity for the long wavelength is $\sim 10^{9} \mathrm{~cm}\left(\mathrm{~Hz}^{0.5}\right) / \mathrm{W}$. From the shape of the curve, we can divide the detectivity into two portions. The detectivity for the short wavelength appears higher. With the increase of bias, the maximum value of the detectivity is at around $0.2 \mathrm{~V}$. Over the maximum point, the detectivity decreases with the increase of the bias due to the large dark current. The detectivity for the long wavelength increases as the bias increases because of the increment of the tunneling photohole current $[32,33]$. The division of the two portions originates obviously from the two mechanisms of the photoholes as discussed in the previous three paragraphs.

\section{Conclusion}

We have successfully demonstrated a QDIP in our preliminary work on Ge QDs devices. In IR responsivity property, our QDIPs have a very broad and tunable spectrum of 1.6-20 $\mu \mathrm{m}$ under applied biases. This is very useful to apply these QDIPs in thermal image detection. For detectivity, the maximum single wavelength detectivity of our devices is about $2.1 \times 10^{10} \mathrm{~cm}\left(\mathrm{~Hz}^{0.5}\right) / \mathrm{W}$ under $0.2 \mathrm{~V}$ for $6 \mu \mathrm{m}$ wavelength at $30 \mathrm{~K}$. The $I-V$ characteristics shows that the dark current is reduced by the thick Si blocking layers so that the detector can be applied in the thermal image detection. The responsivity at zero bias is around $1.6-9 \mu \mathrm{m}$. We can increase the responsivity from 9 to $20 \mu \mathrm{m}$ with the external bias. The tunability of the responsivity spectrum under the applied bias results from the utility of the two mechanisms of the photoholes to be emitted over or tunnel through the silicon barrier. 


\section{Acknowledgement}

This work was partly supported by the National Science Council of the Republic of China under Contract Number NSC 90-2215-E002-024-.

\section{References}

[1] Gunapala SD, Bandara KMSV, Levine BF, Sarusi G, Park JS, Lin TL, et al. High performance InGaAs/GaAs quantum well infrared photodetectors. Appl Phys Lett 1994;64: 3431-3.

[2] West LC, Eglash SJ. First observation of an extremely large-dipole infrared transition within the conduction band of a GaAs quantum well. Appl Phys Lett 1985;46(12): $1156-8$.

[3] Levine BF, Bethea CG, Hasnain G, Shen VO, Pelve E, Abbott RR, et al. High sensitivity low dark current $10 \mu \mathrm{m}$ GaAs quantum well infrared photodetectors. Appl Phys Lett 1990;56(9):851-3.

[4] Levine BF, Gunapala SD, Kuo JM, Pei SS, Hui S. Normal incidence hole intersubband absorption long wavelength GaAs/ $/ \mathrm{Al}_{x} \mathrm{Ga}_{1-x}$ As quantum well infrared photodetectors. Appl Phys Lett 1991;59(15):1864-6.

[5] Levine BF. Quantum-well infrared photodetectors. J Appl Phys 1993;74(8):R1-R81.

[6] Bandara KMSV, Levine BF, Sarusi G, Park JS, Lin TL, Pike WT, et al. High performance InGaAs/GaAs quantum well infrared photodetectors. Appl Phys Lett 1994;64(25): 3431-3.

[7] Schneider H, Walther M, Schönbein C, Rehm R, Fleissner J, Pletschen W, Braunstein J, et al. QWIP FPAs for highperformance thermal imaging. Phys E 2000;7(1-2):101-7.

[8] Fromherz T, Mac W, Hesse A, Bauer G, Miesner C, Brunner $\mathrm{K}$, et al. Intraband absorption and photocurrent spectroscopy of self-assembled p-type $\mathrm{Si} / \mathrm{SiGe}$ quantum dots. Appl Phys Lett 2002;80(12):2093-5.

[9] Chen Z, Baklenov O, Kim ET, Mukhametzhanov I, Tie J, Madhukar A, et al. Normal incidence InAs/ $\mathrm{Al}_{x} \mathrm{Ga}_{1-x} \mathrm{As}$ quantum dot infrared photodetectors with undoped active region. J Appl Phys 2001;89(8):4558-63.

[10] Pan D, Towe E, Kennerly S. Normal-incidence intersubband (In, Ga)As/GaAs quantum dot infrared photodetectors. Appl Phys Lett 1998;73(14):1937-9.

[11] Chu L, Zrenner A, Böhm G, Abstreiter G. Normal-incident intersubband photocurrent spectroscopy on InAs/GaAs quantum dots. Appl Phys Lett 1999;75(23):3599-601.

[12] Ryzhii V. Analysis of the photocurrent in quantum dot infrared photodetectors. Jpn J Appl Phys, Part 2: Letters 2001;40(2B):L148-50.

[13] Ryzhii V, Pipa V, Khmyrova I, Mitin V, Willander M. Dark current in quantum dot infrared photodetectors. Jpn J Appl Phys, Part 2: Letters 2000;39(12B):L1283-5.

[14] Wang KL, Karunasiri RPG. SiGe/Si electronics and optoelectronics. J Vac Sci Technol B 1993;11(3):1159-67.

[15] Chen CC, Chen HC, Hsu MC, Kuan CH. Performance and application of a superlattice infrared photodetector with a blocking barrier. J Appl Phys 2002;91:943-8.

[16] Chen CC, Chen HC, Kuan CH, Lin SD, Lee CP. Multicolor infrared detection realized with two distinct super- lattices separated by a blocking barrier. Appl Phys Lett 2002;80:2251-3.

[17] Chen CC, Chen HC, Hsu MC, Hsieh WH, Kuan CH. Relaxation mechanisms of the photoelectrons in the second miniband of a superlattice structure. IEEE $\mathrm{J}$ Quant Electron 2003;39(2):306-13.

[18] Kuan C-H, Hsieh W-H, Lin S-Y, Chen C-C, Chen J-M. Investigation of superlattice infrared photodetectors to reach the background limited performance at high temperature. In: Brown GJ, Razeghi M, editors. Photodetector: materials and devices VI, 2001.

[19] Ryzhii V. Physical model and analysis of quantum dot infrared photodetectors with blocking layer. J Appl Phys 2001;89(9):5117-24.

[20] Ryzhii V. The theory of quantum-dot infrared phototransistors. Semicond Sci Technol 1996;11:759-65.

[21] Wang SY, Lin SD, Wu HW, Lee CP. Low dark current quantum-dot infrared photodetectors with an AlGaAs current blocking layer. Appl Phys Lett 2001;78(8):10235.

[22] Hsu MC, Kuan CH. Multiple-color GaAs/AlGaAs superlattice infrared photodetector controlled by the polarity and magnitude of the bias voltage. Int Electron Dev Meeting Tech Dig 1999;5:591-4.

[23] Eaglesham DJ, Cerullo M. Dislocation-free StranskiKrastanow growth of $\mathrm{Ge}$ on $\mathrm{Si}(100)$. Phys Rev Lett 1990;64(16):1943-6.

[24] Rodrigues PAM, Cerdeira F, Bean JC. Comment on "Germanium dots with highly uniform size distribution grown on $\mathrm{Si}(100)$ substrate by molecular beam epitaxy". Appl Phys Lett 1999;75(1):145-6 [Appl. Phys. Lett. 71 (1997) 3543].

[25] Dashiell MW, Denker U, Schmidt OG. Photoluminescence investigation of phononless radiative recombination and thermal-stability of germanium hut clusters on silicon (001). Appl Phys Lett 2001;79(14):2261-3.

[26] Schmidt OG, Eberl K, Rau Y. Strain and band-edge alignment in single and multiple layers of self-assembled Ge/ $\mathrm{Si}$ and GeSi/Si islands. Phys Rev B 2000;62(24):1671520.

[27] Fukatsu S, Sunamura H, Shiraki Y, Komiyama S. Suppression of phonon replica in the radiative recombination of an MBE grown typeII GeSi QDs. Thin Solid Films 1998;321:65-9.

[28] Yakimov AI, Stepina NP, Dvurechenskii AV, Nikiforov $\mathrm{AI}$, Nenashev AV. Interband absorption in charged $\mathrm{Ge} / \mathrm{Si}$ type-II quantum dots. Phys Rev B, YEAR 63045312.

[29] Pan D, Towe E. Photovoltaic quantum-dot infrared detectors. Steve Kennerly Appl Phys Lett 2000;76(22): 3301-3

[30] Liu HC, Federico Capasso CA, editors. Intersubband transitions in quantum wells: physics and device application I V62. San Diego: Academic; 2000.

[31] Yakimov AI, Dvurechenskii AV, Nikiforov AI, Proskuryakov YU. Interlevel Ge/Si quantum dot infrared photodetector. J Appl Phys 2001;89(10):5676-81.

[32] Miesner C, Röthig O, Brunner K, Abstreiter G. Midinfrared photocurrent measurements on self-assembled Ge dots in Si. Phys E 2000;7:146-50.

[33] Levine BF. Device physics of quantum well infrared photodetectors. Semicond Sci Technol 1993;8:S400-5. 